\title{
Shift-invariant cone-beam reconstruction outside R-lines with a disconnected source trajectory
}

\author{
Z. Yu, Student Member, IEEE, F. Noo, Member, IEEE, G. Lauritsch, A. Maier, F. Dennerlein, and J. Hornegger
}

\begin{abstract}
Over the past decade, significant progress has been made in theoretically-exact and stable (TES) cone-beam reconstruction using shift-invariant filtering, provided that Tuy's condition is satisfied. However, these algorithms only allow reconstruction at positions that lie on an $R$-line (an $R$-line is any segment of line that connects two source positions). In 2005, Pack and Noo proposed a formula that allows TES reconstruction outside R-lines using shift-invariant filtering. However, for some disconnected source trajectories, the backprojection and filtering steps for this formula are complex. In this work, we propose a new shift-invariant reconstruction formula that allows less complex TES reconstruction outside $\mathbf{R}$-lines for some disconnected source trajectories. This new formula is validated using computer simulated data from a tent trajectory.
\end{abstract}

\section{INTRODUCTION}

The introduction of multidetector computed tomography drastically increases both temporal and spatial resolutions for both interventional and diagnostic imaging, but it also brings new challenges to theoretically-exact and stable (TES) conebeam $(\mathrm{CB})$ image reconstruction algorithms. These algorithms should allow shift-invariant filtering such that efficient implementation is possible. Over the past decade, significant progress has been achieved in this category of reconstruction algorithms [1], [2], [3], [4], [5], [6], [7]. However, these algorithms only allow reconstruction at positions that lie on an R-line (an R-line is any segment of line that connects two source positions), which represents a strong constraint on the source trajectory. This constraint was relaxed by PackNoo Formula D [8] that allows efficient TES reconstruction outside R-lines. This formula was successfully applied to the saddle [9], [10] and the reverse helix [11]. Note that this formula was based on an important observation (or property) of a reconstruction operator called $\mathcal{K}$ in [8], [10]. Though the constraint is loosened, for some cases such as a disconnected trajectory, the backprojection and filtering steps of PackNoo Formula D can still be complex and computationally expensive.

This work was partially supported by a grant from Siemens Medical Solution USA and by the U.S. National Institutes of Health (NIH) under grants No. R21 EB009168 and R01 EB007236. The concepts presented in this paper are based on research and are not commercially available. Its contents are solely the responsibility of the authors and do not necessarily represent the official views of the NIH. Zhicong Yu is with the Department of Radiology, the University of Utah, Salt Lake City, USA (e-mail: zyu@ucair.med.utah.edu). He is also a Ph.D candidate with the Chair of Pattern Recognition, Friedrich-Alexander-University of Erlangen-Nuremberg, Erlangen, Germany. Frederic Noo is with the Department of Radiology, the University of Utah, Salt Lake City, USA (e-mail: noo@ucair.med.utah.edu). Günter Lauritsch, Andreas Maier and Frank Dennerlein are with Siemens AG, Healthcare Sector, Germany. Joachim Hornegger is with the Chair of Pattern Recognition, Friedrich-Alexander-University of Erlangen-Nuremberg, Erlangen, Germany.
In this work, we present a new observation regarding operator $\mathcal{K}$, and we propose from this observation a new shiftinvariant reconstruction formula that allows less complex TES reconstruction outside R-lines for some disconnected source trajectories. This new formula is validated using computer simulated data from a tent-like trajectory.

\section{PRELIMINARIES}

Let $\underline{x}$ be a point of interest, and let $f(\underline{x})$ denote the linear attenuation coefficient at $\underline{x}$, with the assumption that $f$ is compactly supported. We parameterize the source trajectory by $\lambda$, and refer to a vertex point on it as $\underline{a}(\lambda)$. For convenience, we adopt some of the notation from [8]. Let

$$
g(\lambda, \underline{\alpha})=\int_{-\infty}^{+\infty} d t f(\underline{a}(\lambda)+t \underline{\alpha}),
$$

and let $g^{\prime}(\lambda, \underline{\alpha})$ be its derivative with respect to $\lambda$, where $\underline{\alpha}$ belongs to $3 \mathrm{D}$ unit sphere, which is denoted as $\mathcal{S}^{2}$. Using this notation, we introduce the following quantities, which will be needed in the later sections.

\section{A. Operator $\mathcal{K}$}

This operator, which was first introduced in [5], is defined as follows. Let $\left[\lambda^{-}, \lambda^{+}\right]$identify a connected vertex path, and let $\underline{e} \in \mathcal{S}^{2}$. Then $\mathcal{K}$ is defined as

$$
\mathcal{K}\left(\underline{x}, \underline{e}, \lambda^{-}, \lambda^{+}\right)=-\frac{1}{2 \pi^{2}} \int_{\lambda^{-}}^{\lambda^{+}} d \lambda \frac{g_{F}(\lambda, \underline{x}, \underline{e})}{\|\underline{x}-\underline{a}(\lambda)\|},
$$

where

$$
g_{F}(\lambda, \underline{x}, \underline{e})=f_{-\pi}^{\pi} d \gamma \frac{g^{\prime}(\lambda, \underline{\theta}(\lambda, \underline{x}, \gamma))}{\sin \gamma}
$$

with

$$
\begin{gathered}
\underline{\theta}(\lambda, \underline{x}, \gamma)=\cos \gamma \underline{\alpha}(\lambda, \underline{x})+\sin \gamma \underline{\beta}(\lambda, \underline{x}, \underline{e}), \\
\underline{\alpha}(\lambda, \underline{x})=\frac{\underline{x}-\underline{a}(\lambda)}{\|\underline{x}-\underline{a}(\lambda)\|} \text { and } \underline{\beta}(\lambda, \underline{x}, \underline{e})=\frac{\underline{e}-(\underline{e} \cdot \underline{\alpha}) \underline{\alpha}}{\|\underline{e}-(\underline{e} \cdot \underline{\alpha}) \underline{\alpha}\|} .
\end{gathered}
$$

Note that the integral in Equation 2 is to be understood as a Cauchy Principle value, and $\underline{e}$ identifies the filtering direction.

\section{B. Operator $\mathcal{T}$}

The concept of this operator was first mentioned in [8] and can be considered as a more general form of Operator $\mathcal{K}$. In contrast to $\mathcal{K}$ that deals with a connected vertex path, Operator $\mathcal{T}$ is designed for a pseudo-connected vertex path. Given a point of interest $\underline{x}$, a pseudo-connected vertex path, denoted 
as $\left\{\mathcal{L} \mid \lambda \in\left[\lambda_{s}, \lambda_{e}\right]\right\}$, is defined as a union of $Q$ connected vertex paths, $\left[\lambda_{q}^{-}, \lambda_{q}^{+}\right]$, with $q=1, \ldots, Q$, which satisfies

- $\lambda_{s}=\lambda_{1}^{-}$and $\lambda_{e}=\lambda_{Q}^{+}$,

- $k_{q} \underline{\alpha}\left(\lambda_{q}^{-}, \underline{x}\right)=\underline{\alpha}\left(\lambda_{q-1}^{+}, \underline{x}\right), k_{q}=\{-1,1\}, q=2: Q$,

- $k_{1}=1, r_{Q}=1$ with $r_{q}=\prod_{m=1}^{q} k_{m}$

As an example, a pseudo-connected vertex path that is composed of 4 connected curves is shown in Figure 1. Using this

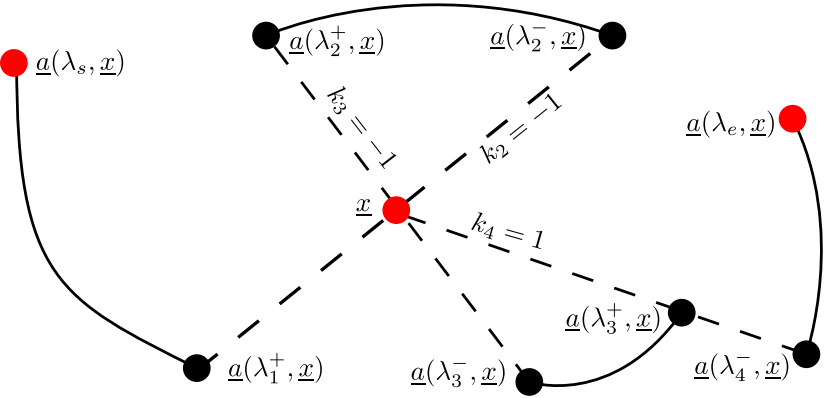

Fig. 1: An example of a pseudo-connected vertex path.

concept of pseudo-connected vertex path, $\mathcal{T}$ can be defined as:

$$
\mathcal{T}\left(\underline{x}, \underline{e}, \lambda_{s}, \lambda_{e}\right)=\sum_{q=1}^{Q} r_{q} \mathcal{K}\left(\underline{x}, \underline{e}, \lambda_{q}^{-}, \lambda_{q}^{+}\right) .
$$

\section{Operator $f_{p}$}

Given a point of interest, $\underline{x}$, and a plane, $\Pi$, that goes through $\underline{x}$ with normal $\underline{n}$, we define $\underline{\alpha}$ as a unit vector in $\Pi$, and refer to $\gamma_{1}$ and $\gamma_{2}$ as two polar angles measured related to $\underline{\alpha}$ in $\Pi$. Let $R(\underline{w}, s)$ be the 3D Radon transform in the plane that is of normal $\underline{w}$ with a signed distance $s$ from the origin along $\underline{w}$. Also, let $R^{\prime \prime}(\underline{w}, s)$ be its second derivative with respect to $s$. Then $f_{p}$ can be defined as below

$f_{p}\left(\gamma_{1}, \gamma_{2} ; \underline{x}, \underline{\alpha}, \underline{n}\right)=-\frac{1}{8 \pi^{2}} \int_{\gamma_{1}}^{\gamma_{2}} d \gamma \int_{0}^{2 \pi} d \theta|\sin \theta| R^{\prime \prime}(\underline{w}, \underline{x} \cdot \underline{w})$,

where

$$
\underline{w}=\sin \theta \cos \gamma \underline{\alpha}+\sin \theta \sin \gamma \underline{\alpha}^{\perp}+\cos \theta \underline{n},
$$

with $\underline{\alpha}^{\perp}=\underline{n} \times \underline{\alpha}$. See Figure 2 for an illustration of notation involved in $f_{p}$. Note that $f_{p}(0, \pi ; \underline{x}, \underline{\alpha}, \underline{n})=f(\underline{x})$.

Because $\mathcal{K}$ is a particular form of $\mathcal{T}$, hereafter we will express everything using $\mathcal{T}$. Also, because $f_{p}$ is fully specified by $\gamma_{1}$ and $\gamma_{2}$, we can analyze $f_{p}$ through the use of 2D drawings in plane $\Pi$ that is orthogonal to $\underline{n}$ and contains $\underline{x}$.

\section{IMPORTANT OBSERVATIONS}

This section is dedicated to two important observations that can be used for development of reconstruction algorithms. The first one was first mentioned in [8] and was further explained in [10]; the second observation is original. These two observations highlight links between Operators $T$ and $f_{p}$. We start with a known result from [5]. Let $\left\{\mathcal{L} \mid \lambda \in\left[\lambda_{A}, \lambda_{B}\right]\right\}$ be a pseudo-connected vertex path, and let $\underline{e} \in \mathcal{S}^{2}$, then

$$
\mathcal{T}\left(\underline{x}, \underline{e}, \lambda_{s}, \lambda_{e}\right)=-\frac{1}{8 \pi^{2}} \int_{\mathcal{S}^{2}} R^{\prime \prime}(\underline{w}, \underline{x} \cdot \underline{w}) \sigma(\underline{x}, \underline{w}, \underline{e}) d \underline{w},
$$

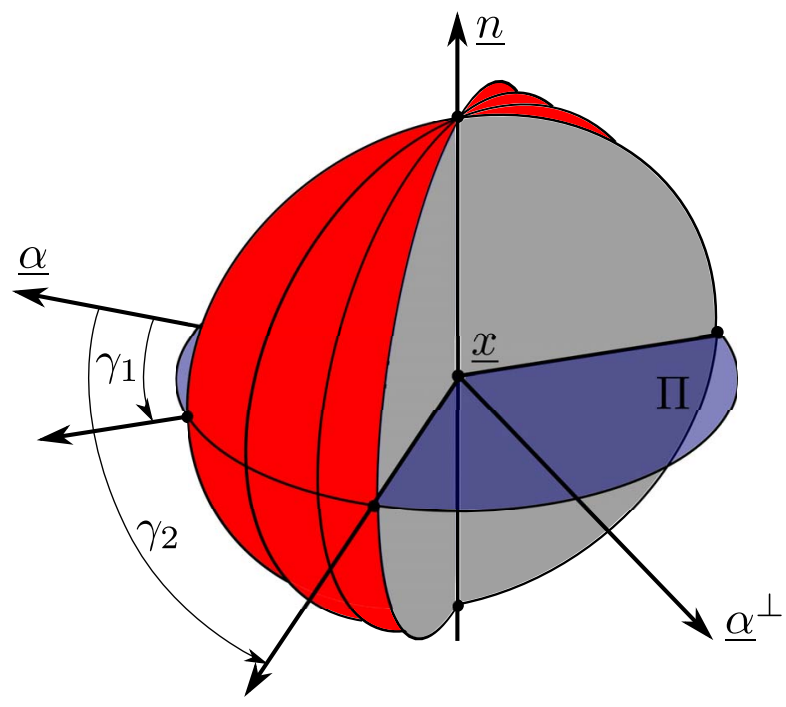

Fig. 2: Illustration for Operator $f_{p}$. Here, $\underline{x}$ is the point of interest, and $\Pi$ is a plane of normal $\underline{n}$ that contains $\underline{x}$. Plane $\Pi$ is spanned by two orthonormal vectors $\underline{\alpha}$ and $\underline{\alpha}^{\perp}$. The spherical surface indicated by red is specified by $\gamma_{1}$ and $\gamma_{2}$, and can be considered as the union of the normal vectors of the Radon planes that contribute to $f_{p}\left(\gamma_{1}, \gamma_{2} ; \underline{x}, \underline{\alpha}, \underline{n}\right)$.

where

$\sigma(\underline{x}, \underline{w}, \underline{e})=\frac{1}{2} \operatorname{sgn}(\underline{w} \cdot \underline{e})\left[\operatorname{sgn}\left(\underline{w} \cdot \underline{\alpha}\left(\lambda_{s}, \underline{x}\right)\right)-\operatorname{sgn}\left(\underline{w} \cdot \underline{\alpha}\left(\lambda_{e}, \underline{x}\right)\right)\right]$.

Next, we introduce some notation. Let $\underline{x}$ be the point of interest, and let $\Pi$ be a plane of normal $\underline{n}$ that goes through $\underline{x}$. This plane intersects a pseudo-connected vertex path at points $A$ and $B$ that are specified by $\lambda_{A}$ and $\lambda_{B}$. For abbreviation, we denote $\underline{\alpha}_{A}$ and $\underline{\alpha}_{B}$ as the unit vectors pointing from $A$ and $B$ to $\underline{x}$, respectively. Furthermore, we define $\underline{\alpha}_{A}^{\perp}=\underline{n} \times \underline{\alpha}_{A}$ and $\underline{\alpha}_{B}^{\perp}=\underline{n} \times \underline{\alpha}_{B}$. Also, we denote $\gamma_{A B}$ as the polar angle from $\underline{\alpha}_{A}$ to $\underline{\alpha}_{B}$, i.e.,

$$
\tan \left(\gamma_{A B}\right)=\underline{\alpha}_{B} \cdot \underline{\alpha}_{A}^{\perp} / \underline{\alpha}_{B} \cdot \underline{\alpha}_{A}, \quad \text { with } \gamma_{A B} \in[-\pi, \pi] .
$$

This notation will be used in the rest of this work, and will be applied to different point labels.

Using this notation and Equation 4, the two observations can be expressed as below.

- Observation I: For a filtering direction $\underline{e}_{0}$ in the fan region that is bounded by $\underline{\alpha}_{A}$ and $-\underline{\alpha}_{B}$, i.e.,

$$
\underline{e}_{0}=\rho \cos \eta \underline{\alpha}_{A}-\rho \sin \eta \underline{\alpha}_{B},
$$

with $\rho>0$ and $\eta \in[0, \pi / 2]$, Operators $\mathcal{T}$ and $f_{p}$ satisfy the following relation

$$
\mathcal{T}\left(\underline{x}, \underline{e}_{0}, \lambda_{A}, \lambda_{B}\right)=\operatorname{sgn}\left(\gamma_{A B}\right) f_{p}\left(0, \gamma_{A B} ; \underline{x}, \alpha \frac{\perp}{A}, \underline{n}\right) .
$$

Illustration of this relation for the case of $\gamma_{A B} \in[0, \pi)$ is on the top of Figure 3. Note that this observation needs only one filtering direction, but it also provides only one choice of $f_{p}$, i.e., the spherical contribution (red region in Figure 3) that is specified by $\underline{\alpha}_{A}^{\perp}$ and $\underline{\alpha}_{B}^{\perp}$. 

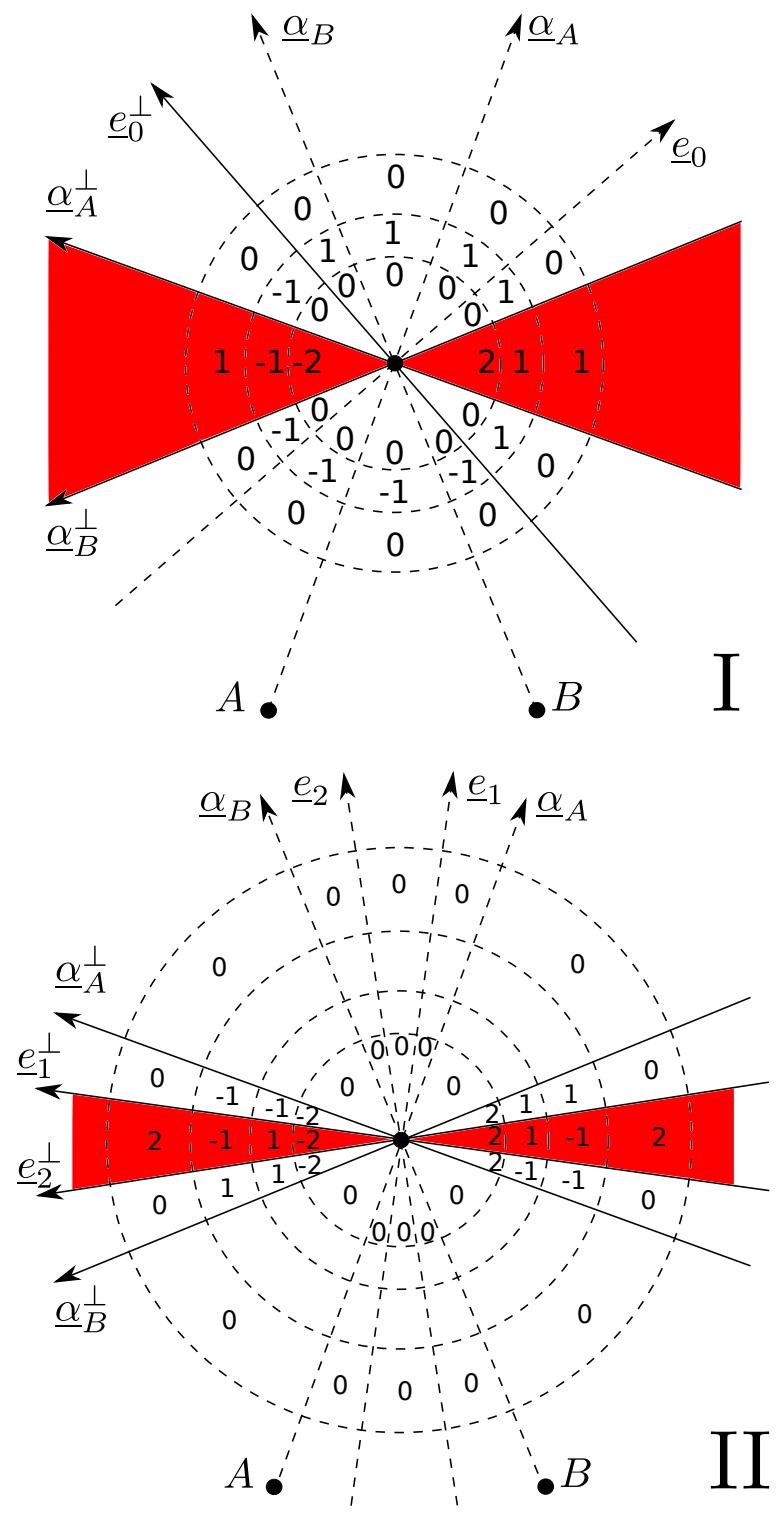

Fig. 3: Illustrations for Observations I and II. We analyze $\sigma(\underline{x}, \underline{w}, \underline{e})$ in plane $\Pi$ using the azimuth angle of $\underline{w}$. The component of $\sigma(\underline{x}, \underline{w}, \underline{e})$ is calculated in differenct rings (or pie). Meaning of the content of the rings (pie), from centremost to outermost. Top: $\operatorname{sgn}\left(\underline{w} \cdot \underline{\alpha}_{A}\right)-\operatorname{sgn}\left(\underline{w} \cdot \underline{\alpha}_{B}\right) ; \operatorname{sgn}\left(\underline{w} \cdot \underline{e}_{0}\right)$; $\sigma\left(\underline{x}, \underline{w}, \underline{e}_{0}\right)$. Bottom: $\operatorname{sgn}\left(\underline{w} \cdot \underline{\alpha}_{A}\right)-\operatorname{sgn}\left(\underline{w} \cdot \underline{\alpha}_{B}\right) ; \sigma\left(\underline{x}, \underline{w}, \underline{e}_{1}\right) ;$ $\sigma\left(\underline{x}, \underline{w}, \underline{e}_{2}\right) ; \sigma\left(\underline{x}, \underline{w}, \underline{e}_{1}\right)-\sigma\left(\underline{x}, \underline{w}, \underline{e}_{2}\right)$. In each picture, the red region indicates the union of the normal vectors of the Radon planes that contribute to $f_{p}$.

- Observation II: For filtering directions $\underline{e}_{1}$ and $\underline{e}_{2}$ in the fan region that is bounded by $\underline{\alpha}_{A}$ and $\underline{\alpha}_{B}$, i.e.,

$\underline{e}_{1}=\cos \gamma_{1} \underline{\alpha}_{A}+\sin \gamma_{1} \underline{\alpha}_{A}^{\perp}, \gamma_{1}=t_{1} \gamma_{A B}$ and $t_{1} \in[0,1]$, $\underline{e}_{2}=\cos \gamma_{2} \underline{\alpha}_{A}+\sin \gamma_{2} \underline{\alpha}_{A}^{\perp}, \gamma_{2}=t_{2} \gamma_{A B}$ and $t_{2} \in[0,1]$,

we have

$$
\begin{aligned}
& \mathcal{T}\left(\underline{x}, \underline{e}_{1}, \lambda_{A}, \lambda_{B}\right)-\mathcal{T}\left(\underline{x}, \underline{e}_{2}, \lambda_{A}, \lambda_{B}\right) \\
& =2 \operatorname{sgn}\left(\gamma_{A B}\right) f_{p}\left(\left[\gamma_{1}, \gamma_{2}\right] ; \underline{x}, \underline{\alpha} \dot{\perp}, \underline{n}\right) .
\end{aligned}
$$

An example of Observation II for the case of $\gamma_{A B} \in$ $[0, \pi)$ is shown on the bottom of Figure 3. In this observation, two filtering directions are needed, however, this offers a freedom to choose $f_{p}$. Note that Observation II is a general form of Observation I, and they become identical when $\underline{e}_{1}=\underline{\alpha}_{A}$ and $\underline{e}_{2}=\underline{\alpha}_{B}$.

Observation II represents the main result of this work. Using Observations I and II, we propose a new reconstruction formula in the next section.

\section{THE NEW RECONSTRUCTION FORMULA}

Let $\Pi$ of normal $\underline{n}$ intersect a pseudo-connected vertex path, $\mathcal{L}$, at $A, C, B$ and $D$ that are specified by $\lambda_{A}, \lambda_{C}, \lambda_{B}$ and $\lambda_{D}$, respectively. $A C B D$ anti-clockwise form a polygon, and $\underline{x}$ is completely inside this polygon. Without loss of generality, we assume $C$ is on the right side of $A B$ viewing from $A$ to $B$, and $D$ is on the left side, as shown in Figure 4 .

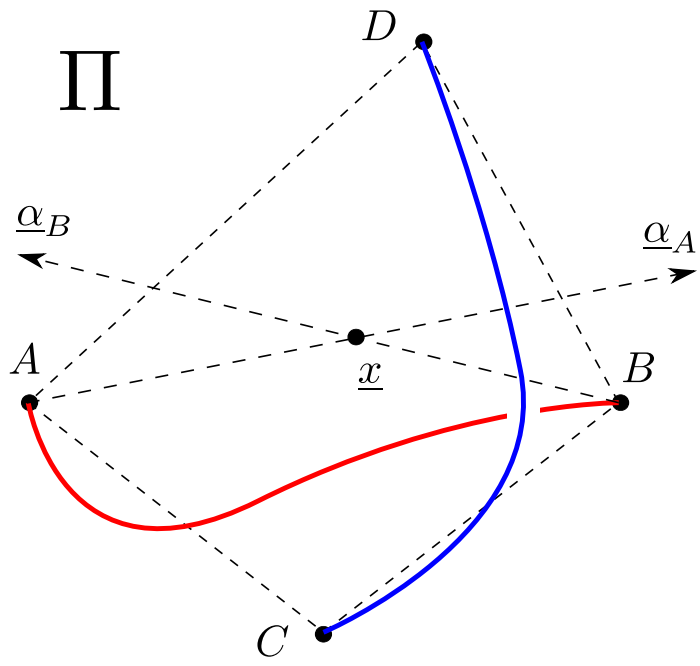

Fig. 4: Illustrations for the new reconstruction formula. The point of intersect, $\underline{x}$, is inside the polygon that is formed by $A, C, B$ and $D$ in $\Pi$. $A B$ and $C D$ are two vertex paths, and they are not necessarily connected to each other.

If $A$ and $B$ are pseudo-connected, and so are $C$ and $D$, then $f(\underline{x})$ can be accurately reconstructed using the following formula.

$$
\begin{aligned}
& f(\underline{x})=\mathcal{T}\left(\underline{x}, \underline{e}_{A B}, \lambda_{A}, \lambda_{B}\right) \\
& +\frac{\operatorname{sgn}\left(\gamma_{A B}\right)}{2}\left(\mathcal{T}\left(\underline{x}, \underline{\alpha}_{A}, \lambda_{C}, \lambda_{D}\right)+\mathcal{T}\left(\underline{x}, \underline{\alpha}_{B}, \lambda_{C}, \lambda_{D}\right)\right),
\end{aligned}
$$

where $\gamma_{A B}$ is defined by Equation (5) and

$$
\underline{e}_{A B}=\rho \cos \eta \underline{\alpha}_{A}-\rho \sin \eta \underline{\alpha}_{B},
$$

with $\rho>0$ and $\eta \in[0, \pi / 2]$. Note that with the new formula, $\underline{x}$ is not necessarily on R-lines, and vertex paths $A B$ and $C D$ are not necessarily connected.

Take the case in Figure 4 as an example. The first term of the right of Equation 6 is identical to $f_{p}\left(0, \gamma_{A B} ; \underline{x}, \underline{\alpha} \frac{\perp}{A}, \underline{n}\right)$ (red region on the top of Figure 5), and the second term contributes to $f_{p}\left(\gamma_{A B}, \pi ; \underline{x}, \underline{\alpha} \frac{\perp}{A}, \underline{n}\right)$ (blue region on the bottom of Figure 5). And by definition of $f_{p}$, the addition on the left and right of Figure 5 exactly provides $f(\underline{x})$. 

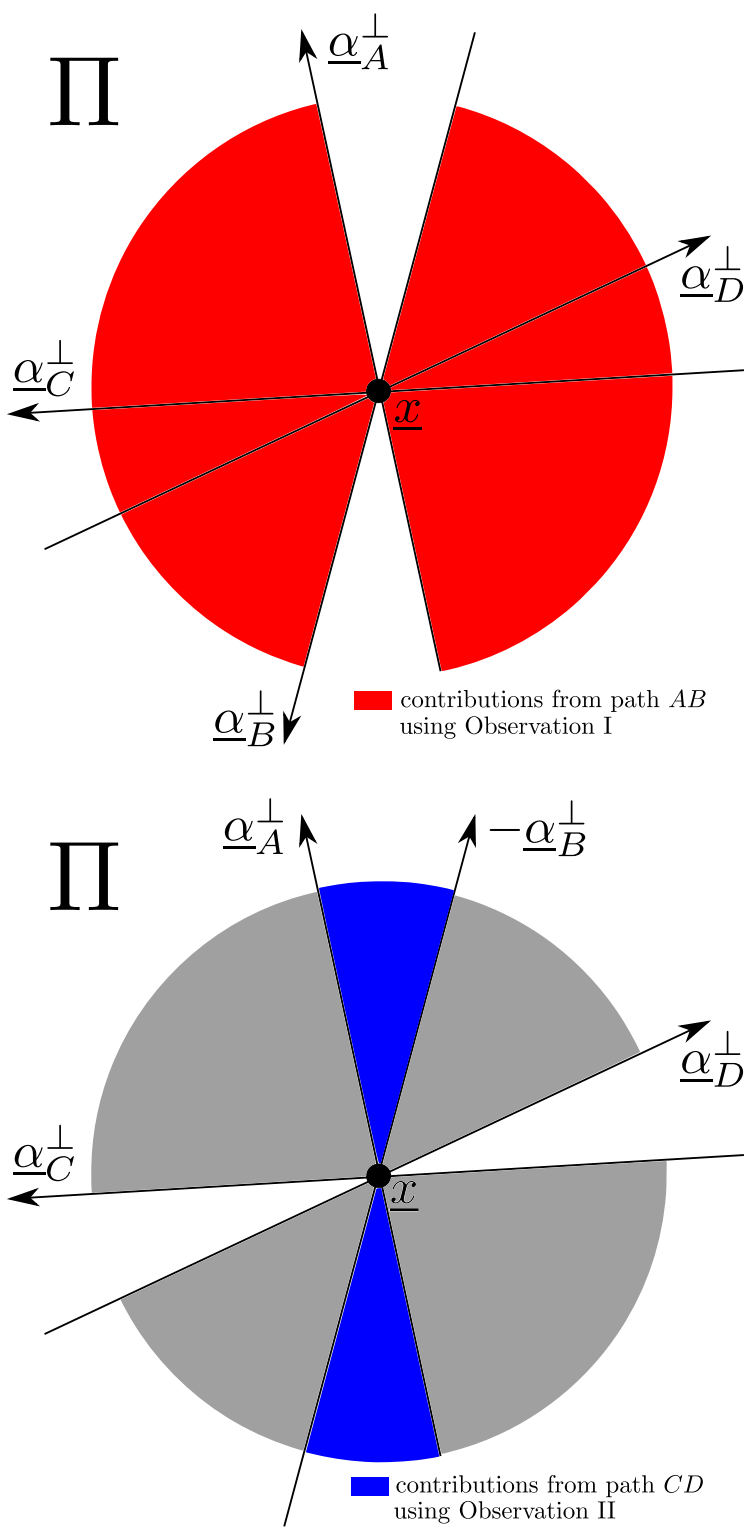

Fig. 5: An insight of the new reconstruction formula for the case presented in Figure 4. We denote a Radon plane by the azimuth angle of its normal vector in plane $\Pi$. Top: red indicates the contribution of the Radon planes from path $A B$ to the reconstruction of $f(\underline{x})$. Bottom: blue indicates the contribution of the Radon planes from path $C D$ to the reconstruction of $f(\underline{x})$, whereas blue and gray together indicate the contribution that path $C D$ is capable of.

\section{COMParison With Pack-Noo Formula D}

The new reconstruction formula is different from Pack-Noo Formula D [8], because it employs both Observations I and II, whereas Pack-Noo Formula D only uses Observation I. We illustrate this difference using an academic example for the source trajectory called the tent trajectory (Figure 6). The tent trajectory consists of two disconnected half circles that are centered on the origin of the $(x, y, z)$ coordinate system. Both half circles are above the $(x, y)$-plane, with one of them in the $(x, z)$-plane with radius $r_{1}$ and the other in the $(y, z)$ - plane with radius $r_{2}$. We assume $0<r_{1}<r_{2}$ such that these two half circles are not connected. Also, we assume that the object can be scanned without data truncation, and completely fit within a sphere that is centered on the origin with a radius smaller than $r_{1}$.

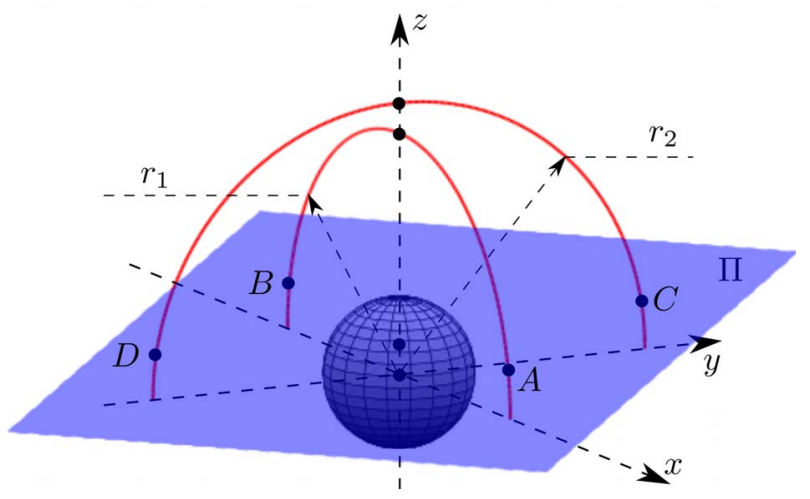

Fig. 6: The tent trajectory is composed of two half circles that are above the $(x, y)$-plane with their centers at the origin of the $(x, y, z)$ coordinate system. One of half circles is located in the $(x, z)$-plane with radius of $r_{1}$, and the other is located in the $(y, z)$-plane with radius of $r_{2}$, where $0<r_{1}<r_{2}$.

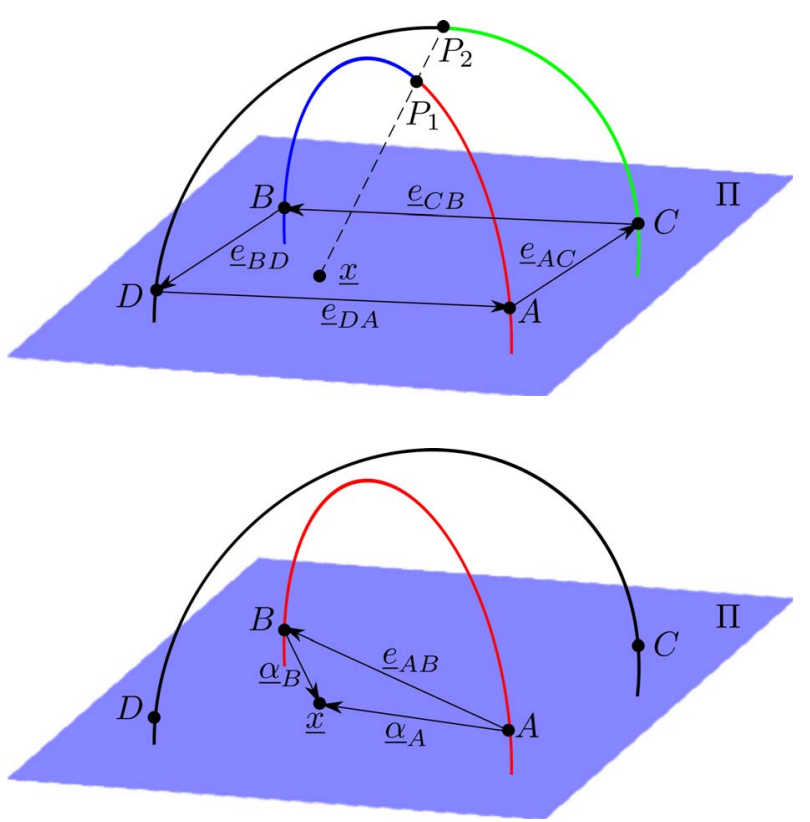

Fig. 7: Top: reconstruction of $\underline{x}$ using Pack-Noo Formula D. Bottom: reconstruction of $\underline{x}$ using the new reconstruction formula. Note that there exist a line through $\underline{x}$ that intersects the half circle in the $(x, z)$-plane at $P_{1}$, and intersects the half circle in the $(y, z)$-plane at $P_{2}$.

Now consider a horizontal plane (perpendicular to the $z$ axis) that intersects the tent trajectory at $A$ and $B$ in the $(x, z)$ plane and $C$ and $D$ in the $(y, z)$-plane. Let $\underline{x}$ be a point of 
interest in this plane. By construction, $A C B D$ form a polygon, and $\underline{x}$ is completely located within this polygon.

According to [8], [10], $\underline{x}$ can be reconstructed using PackNoo Formula $\mathrm{D}$, if there exist pseudo-connected paths from $A$ to $C, C$ to $B, B$ to $D$ and $D$ to $A$, which is true for the tent trajectory. It is not difficult to prove that for the given $\underline{x}$ above, there exist a line through $\underline{x}$ that intersects both half circles. We denote $P_{1}$ and $P_{2}$ as the intersections between this line and the half circles in the $(x, z)$-plane and in the $(y, z)$ plane, respectively. Through $P_{1}$ and $P_{2}, A, C, B$ and $D$ are pseudo connected, and $\underline{x}$ can be reconstructed. Take $A$ and $C$ as an example, they are pseudo connected by $A P_{1}$ and $P_{2} C$. According to Equation 7 in [8], the filtering direction used for this path can be defined as

$$
\underline{e}_{A C}=\rho \cos \eta \underline{\alpha}_{A}-\rho \sin \eta \underline{\alpha}_{C},
$$

with $\rho>0$ and $\eta \in[0, \pi / 2]$. The other three paths can be obtained in a similar way, so do their filtering directions. An illustration is given on the top of Figure 7. Note that $P_{1}$ and $P_{2}$ are $\underline{x}$ dependent, which may increase algorithm complexity and computational cost.

In contrast to Pack-Noo Formula $\mathrm{D}, \underline{x}$ can be reconstructed using the new reconstruction formula without identification of $P_{1}$ and $P_{2}$ (bottom of Figure 7). In this case, only two paths are needed, i.e., $A B$ and $C D$. One filtering direction is needed for path $A B$, i.e., $\underline{e}_{A B}$ defined by Equation 7, whereas two filtering directions are required for path $C D$, i.e., $\underline{\alpha}_{A}$ and $\underline{\alpha}_{B}$.

As described above, for particular situations, the filtering directions used in Pack-Noo Formula $\mathrm{D}$ for paths $A C$ and $C B$ can be identical to $\underline{e}_{A B}$ that is used for path $A B$ in the new reconstruction formula. In this case, for path $A B$, the two formulae become the same. However, for path $C D$, the two formulae are always different. This can be understood by the fact that for path $A B$ both formulae employ Observation I, whereas for path $C D$, the two formulae employ different observations.

\section{Simulation Results}

We conducted numerical simulations using the FORBILD head phantom and the tent trajectory. The two half circles had radii of $r_{1}=80 \mathrm{~cm}$ and $r_{2}=90 \mathrm{~cm}$, and were each sampled 360 vertex points. The source-to-detector distances used for the half circle in the $(x, z)$-plane and in the $(y, z)$-plane were $120 \mathrm{~cm}$ and $135 \mathrm{~cm}$, respectively. The detectors were both composed of $400 \times 400$ square pixels of size $0.1 \mathrm{~cm} \times 0.1 \mathrm{~cm}$ so as to avoid data truncation.

The FORBILD head phantom was reconstructed using a rectangular window in the Hilbert transform step with voxel size of $0.05 \mathrm{~cm} \times 0.05 \mathrm{~cm} \times 0.05 \mathrm{~cm}$. The reconstruction results at $z=0 \mathrm{~cm}$ is illustrated in Figure 8. Note that there is little $\mathrm{R}$-line coverage in this plane. The top image was obtained from path $A B$, and the bottom image was obtained from path $C D$. The middle image represents the addition of the top and bottom contributions. Although the image suffers from discretization errors, the image quality well supports our theoretical result.
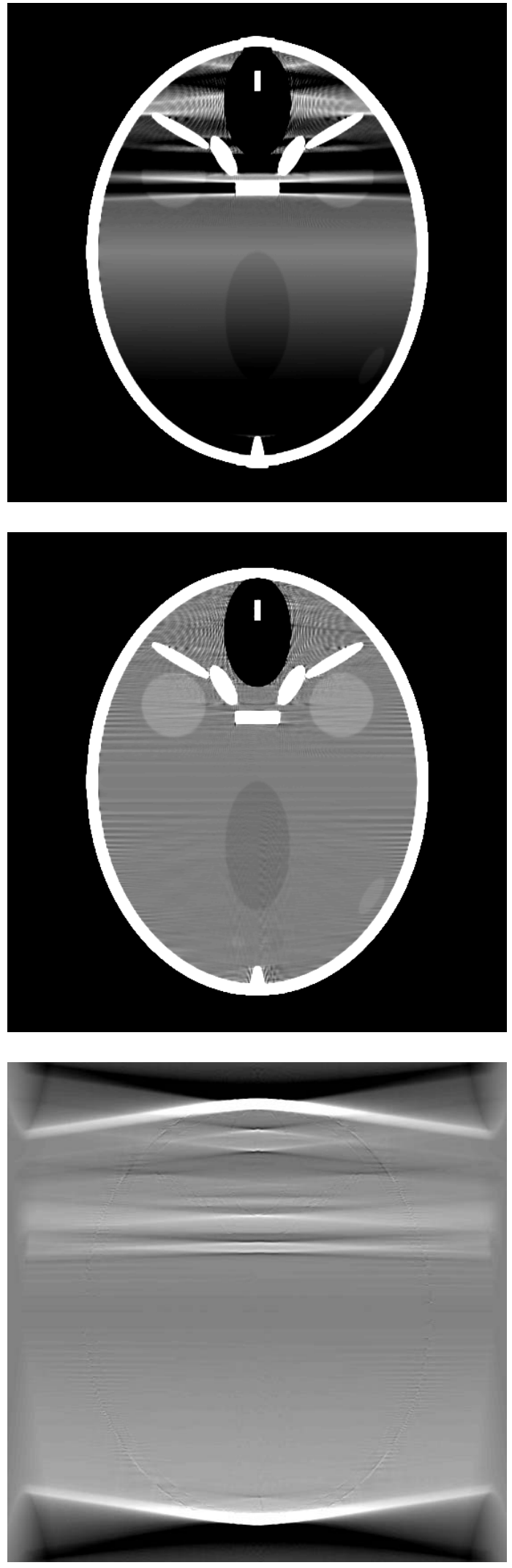

Fig. 8: Reconstruction results for the FORBILD head phantom at $z=0$ using the tent trajectory. Top: the reconstruction obtained from path $A B$. Bottom: the reconstruction from $\mathrm{CD}$. Middle: the addition of results on the top and Bottom. Display window: $[0,100] \mathrm{HU}$. 


\section{CONCLUSIONS}

In this work, we have extended the inversion scheme established by Pack and Noo [8], [5]. First, we have presented two important observations that can be used for developing new TES reconstruction algorithms. Based on these observations, we proposed a new shift-invariant formula that allows TES reconstruction outside R-lines . Using the tent trajectory, we have demonstrated that the new formula employs different filtering directions from that of Pack-Noo Formula D; and that the new formula is less complex than Pack-Noo formula D, because the backprojection step of the latter formula is voxel dependent (identification of $P_{1}$ and $P_{2}$ is required for each point of interest; see Section V). Finally, the new reconstruction formula was validated using computer simulated data from the tent trajectory.

\section{REFERENCES}

[1] A. Katsevich, "Theoretically exact FBP-type inversion algorithm for spiral CT," SIAM J.Appl.Math, vol. 62, pp. 2012-26, 2002.

[2] Y. Zou and X. Pan, "Exact image reconstruction on PI-lines from minimum data in helical cone-beam CT," Phys.Med.Biol., vol. 49, pp. 941-959, February 2004.

[3] J. D. Pack, F. Noo, and R. Clackdoyle, "Cone-beam reconstruction using the backprojection of locally filtered projections," IEEE Trans. Med. Imag., vol. 24, no. 1, pp. 70-85, January 2005.

[4] Y. Ye, S. Zhao, H. Yu, and G. Wang, "A general exact reconstruction for cone-beam ct via backprojection-filtration," IEEE Trans. Med. Imag., vol. 24, no. 9, pp. 1190-1198, 2005.

[5] J. Pack and F. Noo, "Cone-beam reconstruction using 1d filtering along the projection of m-lines," Inverse Problems, vol. 21, pp. 1105-20, 2005.

[6] Y. Zou and X. Pan, "Image reconstruction on pi-lines by use of filtered backprojection in helical cone-beam ct," Phys.Med.Biol., vol. 49, pp. 2717-731, 2004.

[7] Y. Ye and G. Wang, "Filtered backprojection formula for exact image reconstruction from cone-beam data along a general scanning curve," Med. Phys., vol. 32, pp. 42-48, 2005.

[8] J. D. Pack and F. Noo, "Cone-beam reconstruction outside r-lines using the backprojection of 1-d filtered data," in 8th International Meeting on Fully Three-Dimensional Image Reconstruction in Radiology and Nuclear Medicine, Salt Lake City, U.S.A., 2005.

[9] H. Yang, M. Li, K. Koizumi, and H. Kudo, "Exact cone beam reconstruction for a saddle trajectory," Phys.Med.Biol., vol. 51, pp. 1157-72, 2006.

[10] — "Application of pack and noo's cone-beam inversion formula to a wide class of trajectories," in Nuclear Science Symposium Conference Record, 2006. IEEE, vol. 6. IEEE, 2006, pp. 3471-3475.

[11] S. Cho, D. Xia, C. Pellizzari, and X. Pan, "A bpf-fbp tandem algorithm for image reconstruction in reverse helical cone-beam ct," Medical physics, vol. 37, pp. 32-39, 2010. 